\title{
Les sources d'ions négatifs pour le chauffage par injection d'atomes à haute énergie dans les plasmas de fusion
}

Alain Simonin $^{(1)}$ (alain.simonin@(ea.fr), Hubert De-Esch ${ }^{(1)}$, Pascal Garibaldi ${ }^{(1)}$, Christian Grand ${ }^{(1)}$,

Jean-Pierre Boeuf ${ }^{(2)}$, Gwenael Fubiani ${ }^{(2)}$, Gerardus Hagelaar ${ }^{(2)}$

(1) IRFM, CEA Cadarache, 13108 Saint-Paul-lez-Durance Cedex. (2) Laplace, Université Paul Sabatier, 31062 Toulouse Cedex 9

La production d'énergie au

sein d'une centrale de fusion

par confinement magnétique

implique de porter les com-

posants de la réaction (noyaux

de deutérium et de tritium)

à une température de plus

de 100 millions de kelvins.

Pour ce faire, les machines

de fusion (Tokamaks) sont

pourvues de sources d'énergie

auxiliaires, comme l'injection

d'atomes de deutérium à haute

énergie au cour du plasma

chaud.

Le Tokamak ITER, à Cadarache, sera équipé de deux faisceaux d'atomes neutres de deuté-

rium $\left(D^{0}\right) d^{\prime}$ une puissance

de 17 MW à 1 MeV d'énergie, résultant de l'accélération

d'ions négatifs D- produits

au sein de grandes sources

d'ions. La source d'ions négatifs

d'ITER est sans précédent ;

elle fait actuellement l'objet

de travaux de recherche en

France et en Europe.

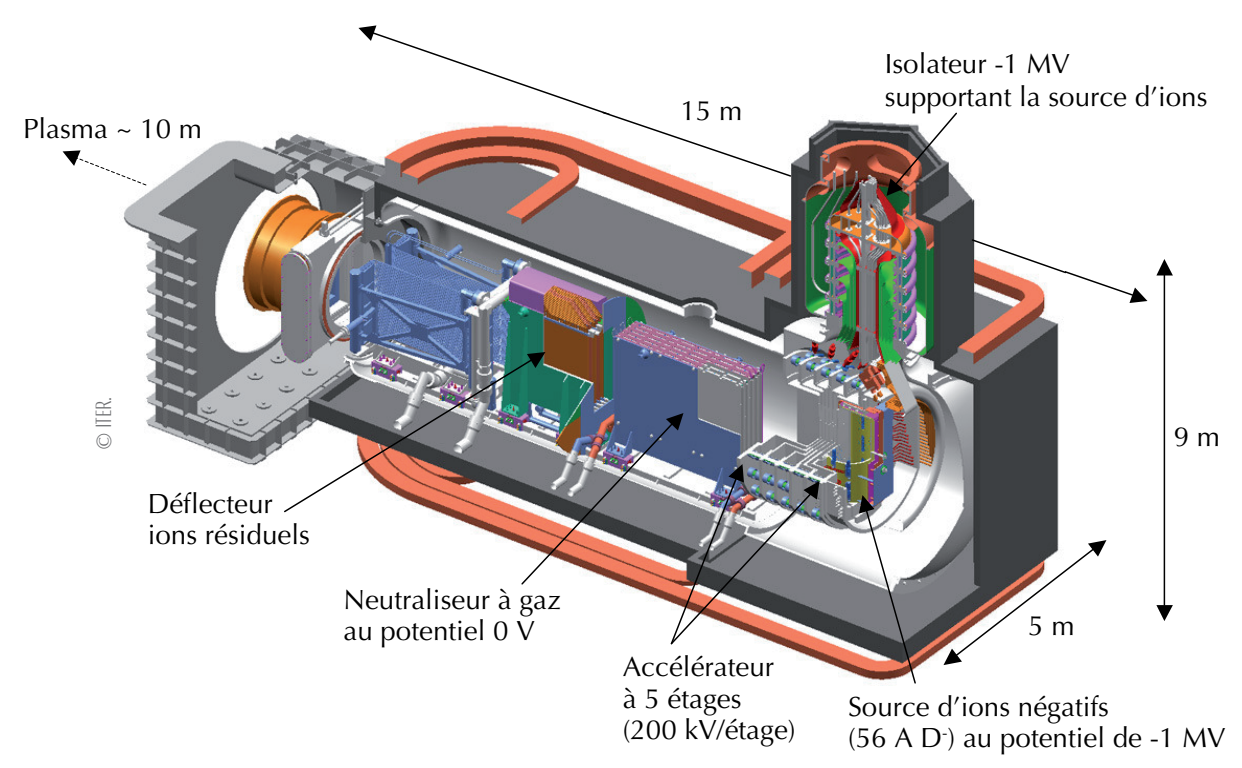

Schéma de la ligne d'injection de neutres d'ITER, comportant deux faisceaux de puissance 17 MW d'atomes neutres de deutérium d'énergie $1 \mathrm{MeV}$.

C'est en 1932, au laboratoire Cavendish de Cambridge, qu'Ernest Rutherford et ses deux collègues (J. Cockcroft et E. Walton) ont mis en évidence la première réaction de fusion, en bombardant une cible de lithium par des protons accélérés à une énergie supérieure à la barrière de potentiel induite par la répulsion coulombienne des noyaux. Dans les machines de fusion actuelles, c'est au cœur d'un plasma de deutérium (D) et de tritium (T) chauffé à plus de 100 millions de kelvins $(\sim 10 \mathrm{keV})$ et confiné magnétiquement dans une enceinte (Tokamak), que se produisent les réactions de fusion [1, 2] (voir encadré, p. 19). Pour atteindre cette température, des sources d'énergie auxiliaires (des systèmes de chauffage additionnel) installées en périphérie du Tokamak, sont en mesure de coupler des dizaines de mégawatts de puissance au foyer thermonucléaire, le centre du plasma, sous forme d'atomes neutres de deutérium $\mathrm{D}^{0}$ à haute énergie ou d'ondes électromagnétiques (voir encadré 1). Ces systèmes de chauffage sont essentiels en phase de démarrage pour l'allumage de la combustion, et dans une moindre mesure pour son contrôle sur de longues durées (plusieurs heures) durant la phase "réacteur». Dans ce mode de fonctionnement, les particules $\alpha$ de 3,5 MeV issues des réactions de fusion D-T prennent en partie le relais des chauffages additionnels, en cédant leur énergie cinétique par collisions aux particules du plasma; atteindre ce fonctionnement " réacteur " est l'étape ultime des recherches et développements autour des machines de fusion. 


\section{Chauffage des plasmas pour l'amorçage des réactions de fusion}

Le courant inductif circulant dans I'anneau de plasma du Tokamak (fig. E1) permet un préchauffage du plasma par effet Joule ; c'est la méthode la plus simple, mais le plasma devenant moins collisionnel (le libre parcours moyen des particules du plasma devient du même ordre de grandeur que ses dimensions caractéristiques) avec l'élévation de température (diminution de sa résistivité), la température ionique sature au-delà de $2 \mathrm{keV}$, bien en dessous du seuil d'amorçage des réactions D-T $(\sim 10 \mathrm{keV})$. Ce chauffage ohmique trouve toutefois son utilité pour démarrer le plasma, puisqu'il assure l'ionisation du gaz et la montée en courant plasma jusqu'à 15 MA pour ITER.

Le chauffage le plus couramment utilisé pour atteindre $10 \mathrm{keV}$ est I'injection d'atomes neutres à haute énergie, dite injection de neutres (IDN), au cœur du plasma (fig. E2). Sur ITER, la méthode consiste à accélérer des ions négatifs de deutérium $\left(D^{-}\right)$à haute énergie $(1 \mathrm{MeV})$ et à les neutraliser (conversion des $\mathrm{D}^{-}$en $\mathrm{D}^{0}$ ) avant leur entrée dans la chambre du Tokamak. Ces atomes neutres sont insensibles au champ magnétique de confinement et pénètrent le plasma sans déflexion, quelles que soient les conditions physiques locales (densité et instabilités du plasma). La densité linéique du plasma est ajustée de façon à ce que ces neutres soient ionisés $\left(D^{0} \rightarrow D^{+}\right)$sous l'effet de collisions, en majorité au cœur du plasma, région où s'effectue le dépôt de puissance (le chauffage) et futur siège des réactions de fusion D-T. Le chauffage du plasma résulte du transfert d'impulsion de ces ions rapides $\left(D^{+}\right.$à $\left.1 \mathrm{MeV}\right)$ piégés le long des lignes de champ magnétique aux particules du plasma $\left(\mathrm{e}^{-}, \mathrm{D}^{+}, \mathrm{T}^{+}\right)$, conduisant notamment à renforcer la partie haute énergie de la fonction de distribution des ions du plasma avec un effet bénéfique sur le taux de réactions de fusion, la section efficace de la réaction D-T étant maximale autour de $100 \mathrm{keV}$.

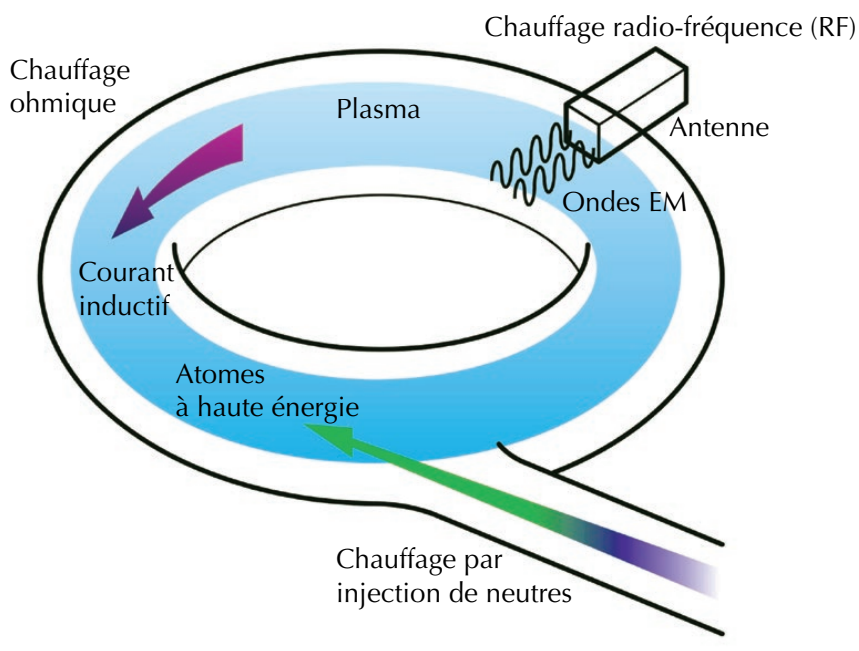

E1. Chauffage additionnel des plasmas.

Chauffage par ondes : le couplage d'une onde électromagnétique (EM) aux particules du plasma se produit lorsque le champ électrique de l'onde est parallèle, en phase, et oscille à la fréquence cyclotron (ionique ou électronique) de la particule le long de la ligne de champ magnétique. Les particules du plasma sont alors accélérées dans la région de couplage et transmettent ensuite leur énergie cinétique au plasma par collisions successives. L'émission de l'onde EM est assurée par des antennes (ou guides d'ondes) radiofréquence en périphérie du plasma (première paroi du Tokamak). Le chauffage par ondes est complémentaire à l'injection de neutres, dans la mesure où il permet des dépôts de puissance très localisés ouvrant la voie à un large éventail de scénarios plasmas.

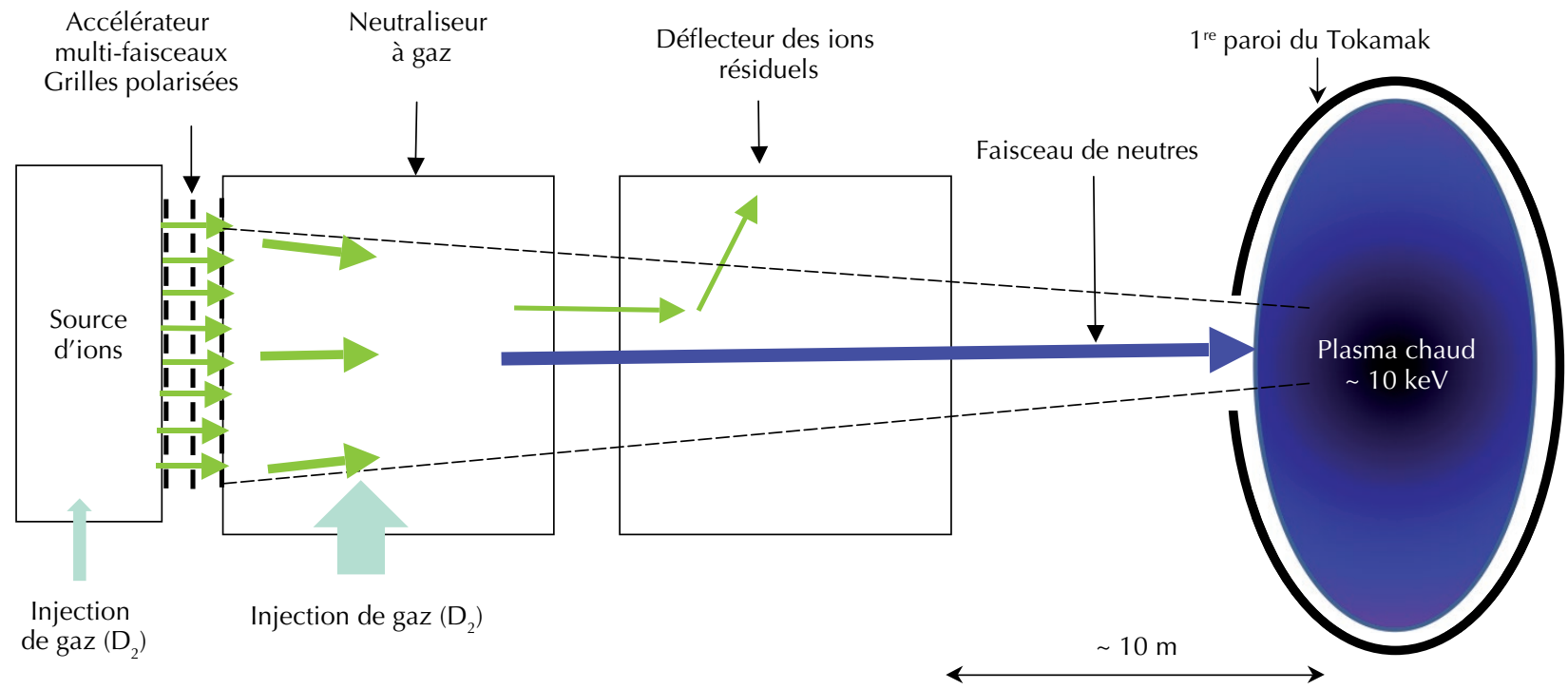

E2. Principe d'une ligne d'injection de neutres. 
>>

Sur ITER, $50 \mathrm{MW}$ de chauffage additionnel seront nécessaires pour initier et produire $500 \mathrm{MW}$ de puissance de fusion D-T pendant 400 secondes au sein du plasma ; c'est un gain en puissance d'un facteur 10, qui reste toutefois insuffisant pour atteindre l'ignition avec production d'électricité.

Le premier démonstrateur de réacteur de fusion (appelé DEMO), visant une puissance de fusion de $2,5 \mathrm{GW}$ pour $1 \mathrm{GW}$ électrique avec un gain en puissance avoisinant 20, pourrait être opérationnel et couplé au réseau à l'aube des années 2050 si l'étape ITER en démontre la faisabilité.

\section{Le chauffage par injection} de neutres (IDN) des machines de fusion par confinement magnétique

L'énergie d'injection de neutres dans les machines actuelles (Tore-Supra au CEA/ Cadarache, JET) avoisine les $100 \mathrm{keV}$; les injecteurs sont basés sur l'accélération d'ions positifs $\left(\mathrm{D}^{+}\right)$, avec neutralisation par échange de charges avec des molécules de deutérium : $\mathrm{D}_{\mathrm{f}}^{+}+\mathrm{D}_{2} \rightarrow \mathrm{D}_{\mathrm{f}}^{0}+\mathrm{D}_{2}{ }^{+}$, où " $\mathrm{D}_{\mathrm{f}}^{+}$" désigne l'ion à haute énergie.

Par exemple, les $16 \mathrm{MW}$ de puissance de fusion D-T produits dans la machine JET en 1998, résultent du chauffage par faisceaux de neutres (deutérium et tritium) injectés simultanément via deux lignes distinctes. La puissance injectée était de $12 \mathrm{MW}$ de $\mathrm{D}^{0}$ à $85 \mathrm{keV}$ et de $9 \mathrm{MW}$ de $\mathrm{T}^{0}$ à $155 \mathrm{keV}$.
Pour ITER, l'augmentation du volume du plasma d'un facteur 8,4 par rapport au JET implique d'accroître à la fois l'énergie et la puissance des faisceaux de neutres (deux faisceaux de $17 \mathrm{MW}$ de $\mathrm{D}^{0}$ à $1 \mathrm{MeV}$ sont prévus). À ce niveau d'énergie, le rendement de neutralisation par échange de charges d'ions $\mathrm{D}^{+}$est quasi nul, alors qu'il reste élevé ( $55 \%$ à $1 \mathrm{MeV}$ ) pour des ions négatifs $\mathrm{D}^{-}$(fig. 1) en raison de la faible énergie de liaison de l'électron à l'atome ( 0,6 eV). Sur l'injecteur d'ITER (voir la figure p. 15), la neutralisation se fera très simplement par collisions avec des molécules de deutérium $\left(\mathrm{D}_{\mathrm{f}}^{-}+\mathrm{D}_{2} \rightarrow \mathrm{D}_{\mathrm{f}}^{0}+\ldots\right)$, suite à une injection de gaz $\mathrm{D}_{2}$ dans le neutraliseur.

En revanche, la relative fragilité de l'ion négatif n'est pas sans inconvénient pour la source et l'accélérateur d'ITER : la densité de courant $\mathrm{D}^{-}$produite dans le plasma de la source $\left(\mathrm{J}_{\mathrm{D}^{-}} \sim 250 \mathrm{~A} / \mathrm{m}^{2}\right)$ est inférieure d'un facteur six à celle des injecteurs actuels à base d'ions positifs $\left(\mathrm{J}_{\mathrm{D}}+\sim 1500 \mathrm{~A} / \mathrm{m}^{2}\right)$ et, dans l'accélérateur, le gaz résiduel diffusant de la source et du neutraliseur conduit à $\sim 28 \%$ de pertes (par neutralisation) des $\mathrm{D}^{-}$en cours d'accélération [3].

Finalement, l'obtention d'un faisceau de $17 \mathrm{MW}(17 \mathrm{~A})$ de $\mathrm{D}^{0}$ à $1 \mathrm{MeV}$ à l'entrée du plasma d'ITER impose des contraintes fortes à la source d'ions négatifs [4] :

i) compte tenu des pertes en ligne de $\mathrm{D}^{-}$et d'un rendement de neutralisation de $55 \%$, la source doit produire $56 \mathrm{~A}_{\text {de }} \mathrm{D}^{-}$à l'entrée de l'accélérateur (au niveau de la surface d'extraction); ii) la densité de courant $\mathrm{D}^{-}$limitée à $\mathrm{J}_{\mathrm{D}^{-}}=250 \mathrm{~A} / \mathrm{m}^{2}$ impose une grande surface d'extraction $\mathrm{S}_{\mathrm{acc}} \sim 1,2 \mathrm{~m}^{2}$ (la surface utile est de $20 \%$ ) et un volume plasma de la source de l'ordre de $0,5 \mathrm{~m}^{3}$;

iii) la production de $\mathrm{D}^{-}$doit être homogène à $\pm 10 \%$ sur toute la surface d'extraction pour conserver une faible divergence du faisceau et une bonne transmission le long de la ligne ;

iv) la source doit fonctionner à basse pression $\left(\mathrm{p}_{\text {source }}<0,3 \mathrm{~Pa}\right.$ ) pour minimiser les pertes des ions négatifs (par neutralisation) dans les étages d'accélération.

C'est une source d'ions négatifs sans précédent qui doit être développée pour ITER ; elle est la clé de voûte de l'injecteur, dont les performances impacteront la frabilité du système dans son ensemble. Des travaux de R\&D (modélisation, expériences) en accompagnement à son développement technologique sont essentiels pour son optimisation future.

\section{La source d'ions négatifs d'ITER}

La source d'ions négatifs (fig. 2) est ellemême un plasma, mais dont les propriétés s'apparentent davantage à celles des plasmas (dits « froids ») utilisés par exemple en microélectronique pour le dépôt de couches minces ou la gravure de semi-conducteurs, qu'au plasma chaud d'ITER. Dans ce type de plasma, les ions négatifs sont produits par bombardement d'une surface métallique par des atomes et ions produits dans la

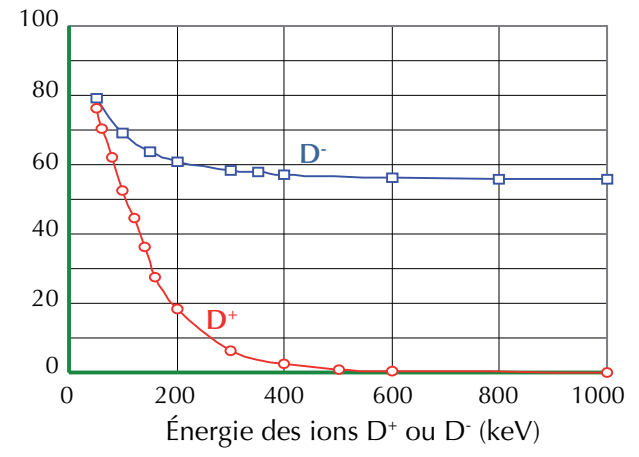

1. Taux de neutralisation (pourcents) des ions $\mathrm{D}^{+}$ et $D^{-}$en fonction de leur énergie.

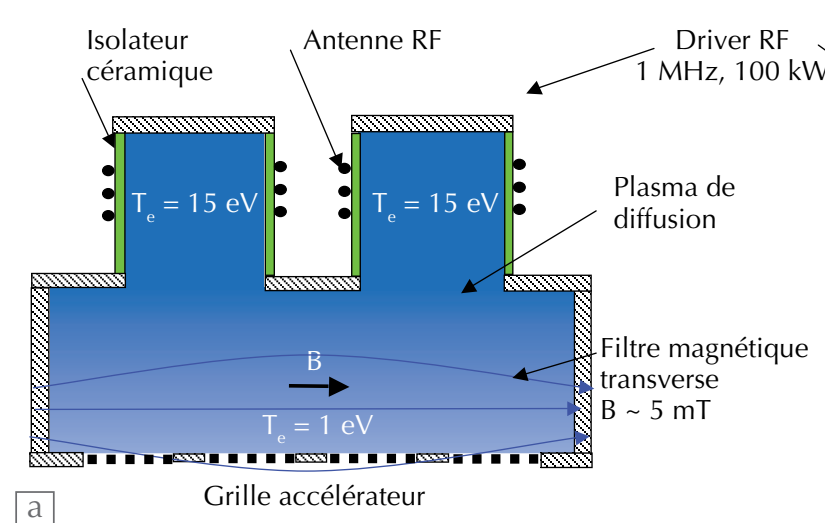

2. La source d'ions négatifs d'ITER.

(a) Schéma de principe de la source (coupe dans le plan horizontal). (b) Dessin en vue arrière de la source. 
$>>$

source. La conversion $\mathrm{D}^{0}\left(\right.$ ou $\left.\mathrm{D}^{+}\right)$en $\mathrm{D}^{-}$ résulte de la capture d'électrons faiblement liés dans le métal, dont le travail de sortie a été abaissé par le dépôt d'un métal alcalin. C'est le césium qui, à ce jour, permet d'atteindre le plus haut rendement de formation de $\mathrm{D}^{-}$; idéalement, une monocouche de césium permet l'abaissement du travail de sortie du métal (cuivre ou tungstène) de typiquement $5 \mathrm{eV}$ à moins de 2,2 eV.

Dans la source d'ITER, c'est la grille métallique, interface entre le plasma source et l'accélérateur, qui sera couverte de césium et soumise au bombardement plasma ; cette production locale permet d'extraire rapidement les ions négatifs vers l'accélérateur et de minimiser leur destruction par collisions avec les particules du plasma (électrons et ions).

Il en ressort deux conditions à satisfaire pour la source d'ions négatifs d'ITER : d'une part produire un plasma à basse pression de deutérium, dense, homogène, à fort taux de dissociation $\left(\mathrm{D}^{0}\right.$ et $\mathrm{D}^{+}$ majoritaires), et, d'autre part, contrôler le dépôt de césium sur la grande surface métallique de l'accélérateur $\left(\mathrm{S}_{\mathrm{acc}} \sim 1,2 \mathrm{~m}^{2}\right)$ soumise au bombardement du plasma.

La source d'ions négatifs d'ITER comporte deux régions plasma (fig. 2), dont les propriétés sont très différentes.
À l'arrière, se trouve la région où le plasma est produit par huit générateurs radiofréquence (RF) $(1 \mathrm{MHz}, 100 \mathrm{~kW}$ par générateur) à couplage inductif, dits "drivers". Chaque driver est l'équivalent d'un transformateur : le primaire est constitué de l'antenne extérieure parcourue par le courant RF, et le secondaire est le courant d'électrons dans le plasma, accélérés par le champ électrique RF induit par l'antenne, qui ionisent le gaz (deutérium). La température électronique $\mathrm{T}_{\mathrm{e}}$ dans le driver est de l'ordre de $15 \mathrm{eV}$ pour une densité du plasma de quelques $10^{18} \mathrm{~m}^{-3}$. La température ionique est beaucoup plus faible $\left(\mathrm{T}_{\mathrm{i}}<2 \mathrm{eV}\right)$, et le degré d'ionisation (pourcentage de gaz ionisé) est également faible, de l'ordre de $10 \%$.

En avant de la source, c'est la région de formation des ions négatifs sous l'effet du bombardement de la surface métallique de l'accélérateur (recouverte du dépôt de césium) par le plasma. La température électronique dans cette région doit être faible $\left(\mathrm{T}_{\mathrm{e}}<1 \mathrm{eV}\right)$, pour éviter un taux de destruction important des ions $\mathrm{D}^{-}$(d'énergie de liaison $\sim 0,6 \mathrm{eV}$ ). Dans la source ITER, c'est un champ magnétique $\mathbf{B}$ transverse (parallèle à la grille d'extraction), de l'ordre de $5 \mathrm{mT}$, qui agit comme un filtre en ralentissant le transport électronique vers la région d'extraction. L'augmentation du temps de séjour des électrons dans le filtre a pour conséquence une augmentation du nombre de collisions électron-neutre, donc des pertes d'énergie électronique, et une réduction significative de la température électronique (de $15 \mathrm{eV}$ dans les drivers à $1 \mathrm{eV}$ dans la région d'extraction).

Les travaux de modélisation de la source ITER [5], où un prototype de source d'ions à un driver a été simulé (fig. 3a), mettent en évidence une dérive des électrons dans la direction $\mathrm{J}_{\mathrm{e}} \times \mathrm{B}$ à l'intérieur du filtre (où J $\mathrm{J}_{\mathrm{e}}$ est la densité de courant électronique dans la direction driver-grille d'extraction), qui augmente de façon significative le transport des électrons (fig. 3b) et entraîne une non-uniformité du plasma dans la région d'extraction (fig. 3c) ; cet effet a été également observé expérimentalement [5], confirmant en partie la validité des modèles numériques. Des travaux de R\&D conciliant modélisation et expériences sont en cours dans différents laboratoires, en vue d'optimiser la topologie du filtre magnétique transverse ou d'explorer d'autres concepts [6]. Le développement d'un modèle 3D massivement parallèle (compatible avec des supercalculateurs) est actuellement en projet, afin de simuler et étudier le comportement du plasma dans la source ITER avec ses 8 drivers (100 kW/driver).

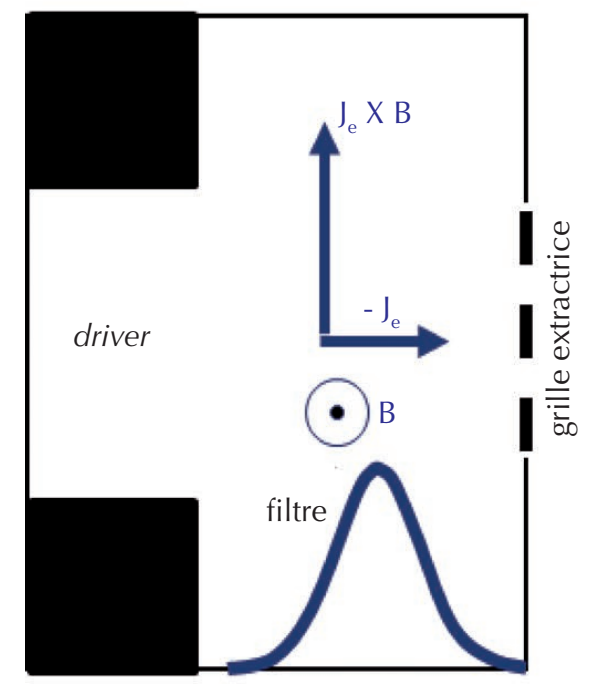

a

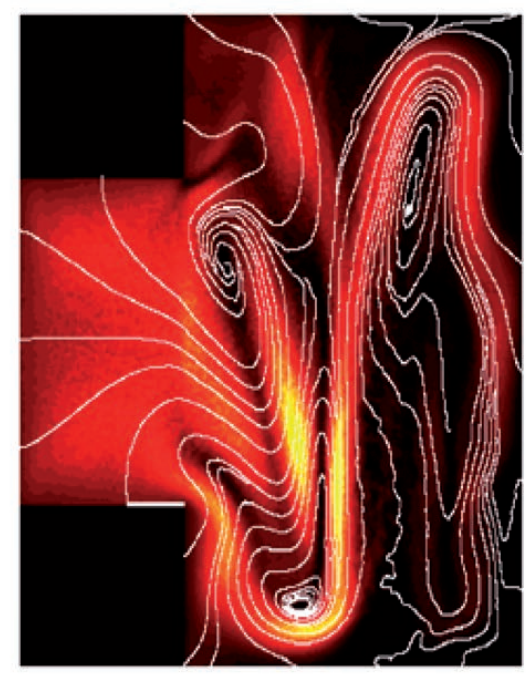

b

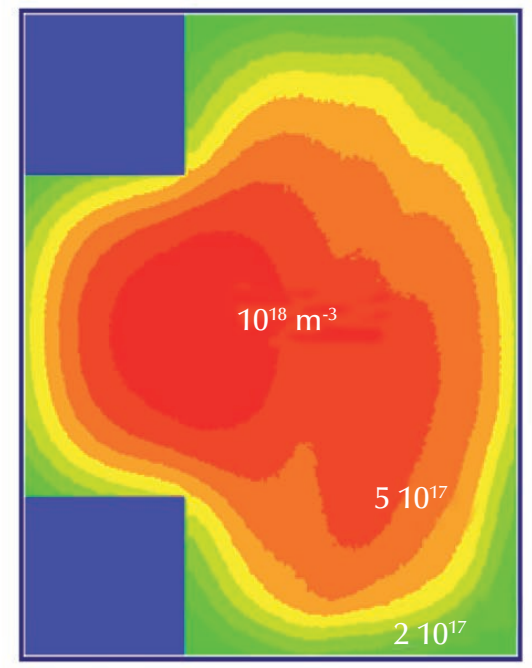

C

3. Modélisation particulaire (PIC MCC : Particle-in-Cell Monte Carlo Collisions) de la source d’ions négatifs.

(a) Domaine de modélisation (le champ magnétique $\mathbf{B}$ du filtre est perpendiculaire au plan de simulation).

(b) Distribution de la densité $\mathbf{J}_{\mathbf{e}}$ de courant électronique dans la source, dérive diamagnétique du courant.

(c) Distribution de la densité de plasma, montrant l'inhomogénéité induite par le filtre magnétique. 


\section{Organisation de la RED autour de la source d'ions négatifs d'ITER}

À ce jour, il n'existe aucune source ayant atteint l'ensemble des spécifications nécessaires pour ITER. Des sources prototypes à échelle réduite sont en cours d'étude dans divers laboratoires, en Europe et dans le monde (Japon, Inde). C'est en Italie (Consortium RFX, Padoue) que deux bancs de test IDN à l'échelle un [7] pour ITER sont en cours de construction, sous la gouvernance du programme cadre européen «Fusion for Energy ( Euratom) : le banc SPIDER, pour le développement et l'optimisation de la source d'ions négatifs (avec huit drivers RF), devrait être opérationnel en 2015-2016 ; pour le banc MITICA, qui permettra d'étudier et optimiser le système dans son ensemble (source, accélérateur, neutraliseur, etc.) avec une ligne IDN à $1 \mathrm{MeV}$ identique à celle d'ITER, les premiers faisceaux de $\mathrm{D}^{0}$ sont attendus en 2017.

C'est en Allemagne, à l'IPP Garching [8], que l'expérimentation sur la source RF pour ITER est la plus avancée, avec une source à l'échelle $1 / 2$ d'ITER (source ELISE avec quatre drivers RF). L'expérimentation sur la source est en cours; à ce jour, elle n'a pas encore atteint les spécifications d'ITER (densité de courant $\mathrm{D}^{-}$, homogénéité, fonctionnement à basse pression, etc.). En France, le CEA et le CNRS sont impliqués, via la «Fédération de Recherche sur la Fusion par Confinement Magnétique » (FR-FCM), dans la R\&D autour du système IDN d'ITER dans son ensemble (source plasma, formation d'ions négatifs, accélérateur, neutraliseur, tenue à très haute tension sous vide). C'est une recherche d'accompagnement, en étroite collaboration avec les partenaires européens (IPP Garching, RFX Padoue), qui vise à développer des modèles physiques (plasma froid magnétisé de la source, de l'accéléra-

\section{Références}

1- J. Jacquinot, Reflets de la physique 32 (2013) 19-25.

2- G. Laval et al., La Fusion Nucléaire : de la recherche fondamentale à la production d'énergie ?,

Rapport scientifique et technique n²6 de l'Académie des sciences, EDP Sciences (2007).

3- G. Fubiani et al., Physical Review Special Topics Accelerators and beams 11 (2008) 014202.

4• R.S. Hemsworth et al., Rev. Sci. Inst., 79 (2008) 026109.

5• J.P. Boeuf et al., Phys. Plasmas 19 (2012) 113510. teur et du neutraliseur), des prototypes de sources en alternative à la source ITER, ou encore, à étudier et explorer de nouveaux concepts sur la formation des ions négatifs sans l'utilisation de césium dans le plasma.

\section{Un système IDN à très haute efficacité pour le réacteur de fusion DEMO}

La recherche actuelle autour des systèmes d'injection de neutres ne se limite pas à ITER. Un réacteur de fusion (DEMO) nécessitera des puissances de faisceaux de neutres $\mathrm{D}^{0}$ de l'ordre de $100 \mathrm{MW}$ avec des énergies d'injection de 1 à $1,5 \mathrm{MeV}$, et un fonctionnement en quasi continu (durée des tirs de plusieurs heures). Le saut technologique et scientifique est conséquent pour DEMO car, en plus de l'augmentation de la puissance, de l'énergie et de la durée de fonctionnement, le système IDN devra avoir un rendement très élevé $\left(\mathrm{P}_{\text {neutres }} / \mathrm{P}_{\text {électrique consommée }}>60 \%\right)$ pour minimiser la puissance électrique consommée par la centrale [9].

Le rendement global de l'injecteur d'ITER étant assez faible ( 30\%), en raison de la neutralisation du faisceau par cible gazeuse limitée à $55 \%$ et des pertes des ions $\mathrm{D}^{-}$ dans l'accélérateur, la R\&D actuelle en France porte sur d'autres méthodes de neutralisation plus efficaces du faisceau : la photoneutralisation des $\mathrm{D}^{-}$pourrait être une solution très avantageuse car, en plus de supprimer une injection massive de gaz dans la chambre du neutraliseur, le taux de neutralisation pourrait atteindre des valeurs très élevées (> 70\%) avec des flux de photons de plusieurs MW. L'utilisation de cavités Fabry-Perot puissantes (1 à $3 \mathrm{MW}$ de puissance de photons intracavité) pourrait être une méthode envisageable pour DEMO, à condition que les travaux de R\&D actuels en France en démontrent la faisabilité.
6• A. Simonin et al., Nucl. Fusion 52 (2012) 063003.

7• P. Sonato et al., "Design of the MITICA neutral beam injector", Proceedings of the 24th IAEA Fusion Energy Conference (2012), International Atomic Energy Agency, P.0. Box 100, A-1400 Vienna, Austria

8• U. Fantz et al., Nucl. Fusion 49 (2009) 125007.

9• J. Pamela et al., Fusion Eng. and Design 84 (2009) 194-204.
Rappels sur la réaction de fusion deutériumtritium dans un réacteur

Avant d'entrer dans le rayon d'action des forces nucléaires à très courte portée $\left(\sim 10^{-15} \mathrm{~m}\right)$ et de fusionner, deux noyaux doivent avoir une énergie cinétique suffisante pour franchir la barrière de potentiel induite par leur propre répulsion coulombienne. La hauteur de la barrière augmente avec la charge du noyau, excluant pour un réacteur de fusion sur terre tous les combustibles autres que l'hydrogène et ses deux isotopes ( $\mathrm{D}$ : deutérium et $\mathrm{T}$ : tritium). C'est la réaction D-T qui, a priori, présente un réel potentiel comme source d'énergie future, avec un amorçage à une température du plasma de 110 millions de kelvin (correspondant à une énergie cinétique des noyaux d'environ $10 \mathrm{keV}$ ). Cette réaction est à la portée des machines de fusion magnétique actuelles (Tokamak), et comporte un taux de réaction dix fois supérieur à celui des autres réactions potentielles (D-D, D-He...).

L'énergie de la réaction D-T est libérée sous forme cinétique par un neutron de $14 \mathrm{MeV}$ (correspondant à $80 \%$ de l'énergie thermique, qui est utilisé pour le chauffage du fluide caloporteur circulant dans la paroi du réacteur), et une particule $\alpha$ (noyau $\mathrm{d}$ 'hélium comportant deux protons et deux neutrons) de 3,5 MeV, source d'autochauffage du plasma de fusion.

La production d'énergie par les réactions de fusion dans un plasma de deutériumtritium implique $d^{\prime}$ atteindre certaines conditions physiques au sein du réacteur : c'est le critère de Lawson, qui résulte du bilan de puissance entre sources (réactions de fusion) et pertes $[1,2]$ du réacteur. II s'exprime au travers de trois paramètres : la densité du plasma $n$ (en pratique, on considère que le plasma est composé de deutérium et de tritium en proportions égales), la température ionique du plasma $T_{i}$ et le temps de confinement de l'énergie $\tau_{\mathrm{e}}$ [1]. Numériquement, le critère de Lawson traduit la condition d'auto-entretien des réactions de fusion (les pertes sont compensées par l'énergie produite lors de la fusion D-T). Il est défini par les deux conditions suivantes: $n \tau_{e} \geq 1,510^{20} \mathrm{~m}^{-3}$.s et $T_{i} \geq 10 \mathrm{keV}$, équivalent à une température du plasma de deutérium-tritium de plus de 100 millions de degrés. 